\title{
A case series of endoscopic cyclophotocoagulation with 532-nm laser in Japanese patients with refractory glaucoma
}

\author{
Masaki Tanito $\mathbb{1}^{1,2} \cdot$ Shin-ichi Manabe ${ }^{3} \cdot$ Teruhiko Hamanaka $^{4} \cdot$ Hiroyuki Sato ${ }^{5} \cdot$ Kazuhiko Mori $^{6}$
}

Received: 10 March 2019 / Revised: 7 June 2019 / Accepted: 19 June 2019 / Published online: 22 July 2019

(c) The Author(s), under exclusive licence to The Royal College of Ophthalmologists 2019

\begin{abstract}
Purpose To report an initial case series of Japanese patients with refractory glaucoma treated with endoscopic cyclophotocoagulation (ECP) using an ECP device that was equipped with a 532-nm neodymium-doped yttrium aluminium garnet (Nd:YAG) laser.

Methods This observational case series included 10 consecutive glaucomatous eyes ( 7 with primary open-angle glaucoma; 3 with secondary glaucoma after cataract surgery) of 10 Japanese subjects ( 7 men, 3 women; mean age \pm standard deviation, $65.7 \pm 15.0$ years) who underwent ECP to control intraocular pressure (IOP), and preserve visual function. Age, sex, glaucoma type, ocular surgical history, preoperative and postoperative logarithm of the minimum angle of resolution visual acuity (VA), IOP, number of antiglaucoma medications, perioperative complications, and treatments for complications were collected from the medical and surgical records. The IOP and numbers of antiglaucoma medications were compared between preoperative and postoperative values.

Results The mean preoperative IOP $(27.3 \pm 5.4 \mathrm{~mm} \mathrm{Hg}$; range, 19-36) and number of antiglaucoma medications $(4.4 \pm 0.8$; range, $3-6$ ) decreased significantly by $53 \%$ and $39 \%$ ( $p=0.0005$ and $p=0.0043$, respectively) to $12.9 \pm 5.2 \mathrm{~mm} \mathrm{Hg}$ (range, 8-20) and $2.7 \pm 1.4$ (range, 0-4), respectively, at the final visit. Compared with preoperative values, a mixed-effect regression model showed significant decreases in the IOP and numbers of medications at every time point up to 24 months postoperatively. No phthisis bulbi or persistent hypotony was recorded. At the final visit, compared with preoperative values, the VA decreased in three eyes by $>0.2$ unit because of glaucoma progression.

Conclusions ECP with Nd:YAG laser is a reasonable option in eyes with refractory glaucoma.
\end{abstract}

Supplementary information The online version of this article (https:// doi.org/10.1038/s41433-019-0538-4) contains supplementary material, which is available to authorized users.

Masaki Tanito

tanito-oph@umin.ac.jp

1 Department of Ophthalmology, Shimane University Faculty of Medicine, Izumo, Japan

2 Division of Ophthalmology, Matsue Red Cross Hospital, Matsue, Japan

3 Hayashi Eye Hospital, Fukuoka, Japan

4 Department of Ophthalmology, Japanese Red Cross Medical Center, Tokyo, Japan

5 Satoh Yuya Eye Clinic, Sendai, Japan

6 Department of Ophthalmology, Kyoto Prefectural University of Medicine, Kyoto, Japan

\section{Introduction}

Glaucoma typically is treated medically first to decrease the intraocular pressure (IOP), the only modifiable risk factor for glaucoma development and progression [1]. Further treatment involves surgical procedures such as minimally invasive glaucoma surgery, trabeculotomy, trabeculectomy, or implantation of a glaucoma drainage device $[2,3]$. These procedures are designed to increase aqueous outflow by creating an alternate or improved pathway to Schlemm's canal and the subconjunctival space. In contrast, ciliary body ablation techniques, such as cyclocryotherapy and transscleral cyclophotocoagulation (TSCP), are wellestablished procedures that reduce the IOP by suppressing aqueous production $[4,5]$. Since these early attempts targeted the ciliary epithelium, the most inner part of the ciliary body, from an external approach, the results included under-treatment with no effect or over-treatment with collateral tissue damage because of the absence of direct 
visualization of the target tissue [6]. Most surgeons reserve traditional cyclodestructive procedures for eyes with endstage and refractory glaucoma or eyes with limited visual potential because of the vision-threatening complications of those procedures [6].

$\mathrm{Ab}$ interno ciliary process ablation initially was performed by applying argon laser using an endophotocoagulation probe in eyes that had undergone vitrectomy and lensectomy and allows direct visualization of the ciliary processes by scleral depression [7, 8]. The greatest difficulty with this therapy is that the ciliary processes are difficult to view in vivo except under these unique circumstances [9]. In 1992, a specialized endoscopic device that enables cyclophotocoagulation from inside the eye was developed in the United States [9]. The laser endoscopy console (Endo Optiks, Little Silver, NJ, USA) combines a $175-\mathrm{W}$ xenon light source for illumination, 810-nm diode laser for photocoagulation, helium-neon laser for the aiming beam, and video imaging for intraocular visualization (http://www. endooptiks.com) [6]. The endoscopy probe is available in 19-, 20-, and 23-gauge sizes with fields of view ranging from $70^{\circ}$ to $140^{\circ}$ and depths of focus spanning $1-30 \mathrm{~mm}$ [6]. With this procedure, referred to as endoscopic cyclophotocoagulation (ECP), surgeons can directly coagulate the ciliary epithelium under direct visualization of the ciliary processes. After its development, the efficacy and safety of ECP was tested first in patients with refractory glaucoma and then recently in patients with moderate and mild glaucoma at various sites, most extensively in the United States [10, 11]. In contrast, because of the difficulty in accessing the specialized device, studies of ECP rarely have been conducted in Japan. Here we report an initial case series of Japanese patients with refractory glaucoma treated with ECP using an ECP device that consisted of a regular ophthalmic endoscope and a green laser system.

\section{Subjects and methods}

This observational case series included 10 consecutive glaucomatous eyes of 10 Japanese subjects $(7 \mathrm{men}, 3$ women; mean age \pm standard deviation [SD], $65.7 \pm 15.0$ years) who underwent ECP to control the IOP and preserve visual function at the Matsue Red Cross Hospital between January 2016 and August 2017. The patients were followed for $>1$ year postoperatively. The possible risks and benefits of ECP and other possible glaucoma surgeries/treatments were explained preoperatively to the patients, and the patients who chose ECP were treated with this procedure. The study adhered to the tenets of the Declaration of Helsinki; the institutional review board of Matsue Red Cross Hospital reviewed and approved the research. Preoperatively, all subjects provided written informed consent to undergo surgery and for use of the clinical data regarding the glaucoma treatment obtained during the follow-up periods. The patients' demographic data and surgical procedures are summarized in Table 1.

\section{ECP device and surgical procedure}

The ECP device used in this study is shown in Fig. 1. The main parts of the system are a light source (FL-301, FiberTech Co., Ltd., Chiba, Japan), an observation camera system equipped with an optical filter for avoiding halation during laser oscillation (FC-304F, FiberTech), and a 532$\mathrm{nm}$ neodymium-doped yttrium aluminium garnet $(\mathrm{Nd}$ : YAG) laser system (Novus Spectra, Lumenis Japan Co., Ltd., Tokyo, Japan or Solitaire, Ellex, Inc., Tokyo, Japan) (Fig. 1a). The fibers from these main parts were assembled into a prototypic 20-gauge ECP probe (resolution, 10,000 fibers; angle of view, $70^{\circ}$; FiberTech; Fig. 1b).

One surgeon (MT) performed all surgeries (Fig. 2, Video 1). Standard sub-Tenon anaesthesia was induced using $2 \%$ lidocaine. Two corneal ports were created using a 20-gauge micro-vitreoretinal knife (Mani, Utsunomiya, Japan) at the 2-3 and 9-10 o'clock positions. A viscoelastic material (1\% sodium hyaluronate, Opegan $\mathrm{Hi}$, Santen Pharmaceutical, Osaka, Japan) was injected into the anterior and posterior chambers to maintain the surgical space. Under observation using an endoscope inserted into the anterior chamber via the corneal port (Fig. 2), the laser was oscillated continuously to bleach and shrink the surface of the ciliary processes (Fig. 2). Gas bubble formation and pigment dispersion were regarded as over-coagulation and undesirable. Typically, the laser power and duration were set at $150-200 \mathrm{~mW}$ and $3 \mathrm{~s}$, respectively. Laser ablation was applied through two corneal ports between two thirds and the entire circumference. If the collapsed vitreous was present in the anterior chamber, an anterior vitrectomy was performed using a 23- or 25-gauge vitreous cutter (Alcon Japan Ltd., Tokyo, Japan) through a corneal side port. If the crystalline lens was present, simultaneous phacoemulsification was performed before ECP through a 2.2-mm-wide clear corneal incision created at the right-hand position, and a one-piece soft-acrylic intraocular lens (Vivinex iSert $\mathrm{XY}$ 1, Hoya, Tokyo, Japan) was inserted through the same clear corneal incision. At the end of surgery, $10-15 \mathrm{mg}$ of triamcinolone acetonide (MaQaid Ophthalmic Injection, Wakamoto Pharmaceutical Co., Ltd., Tokyo, Japan) was injected into the sub-Tenon space and $0.3 \%$ ofloxacin ointment (Tarivid, Santen Pharmaceutical) was applied. Finally, $1.5 \%$ levofloxacin (Nipro, Osaka, Japan) and $0.1 \%$ betamethasone (Sanbetason, Santen Pharmaceutical) were applied topically four times daily for 3-4 weeks postoperatively in all cases. 


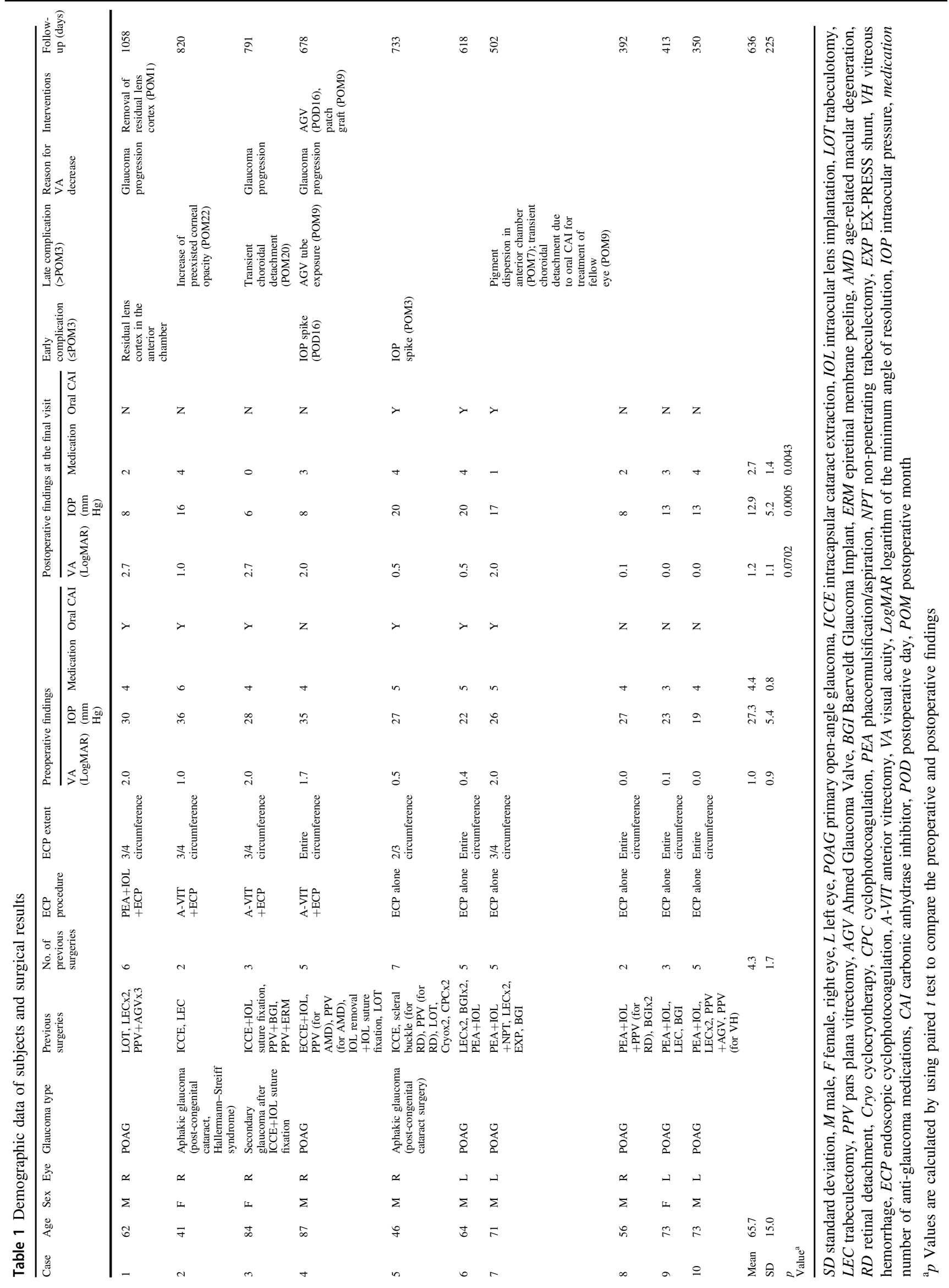




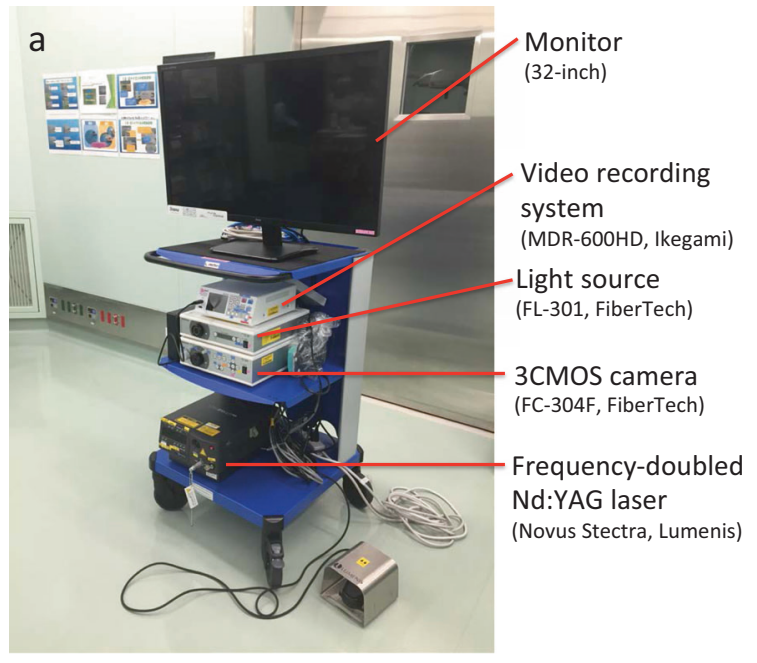

b

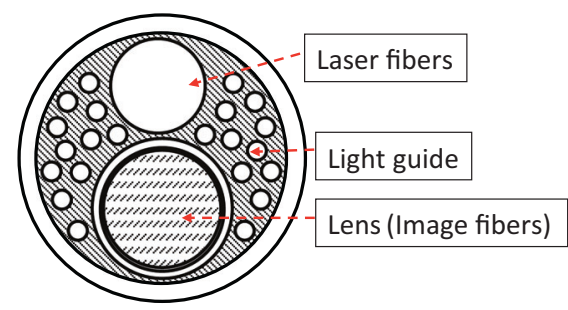

Fig. 1 The developed endoscopic cyclophotocoagulation (ECP) device. a The components of the ECP device. CMOS complementary metal oxide semiconductor, Nd:YAG neodymium-doped yttrium aluminium garnet. b Schematic drawing of the cross-section of the ECP probe (diameter, 20 gauge)
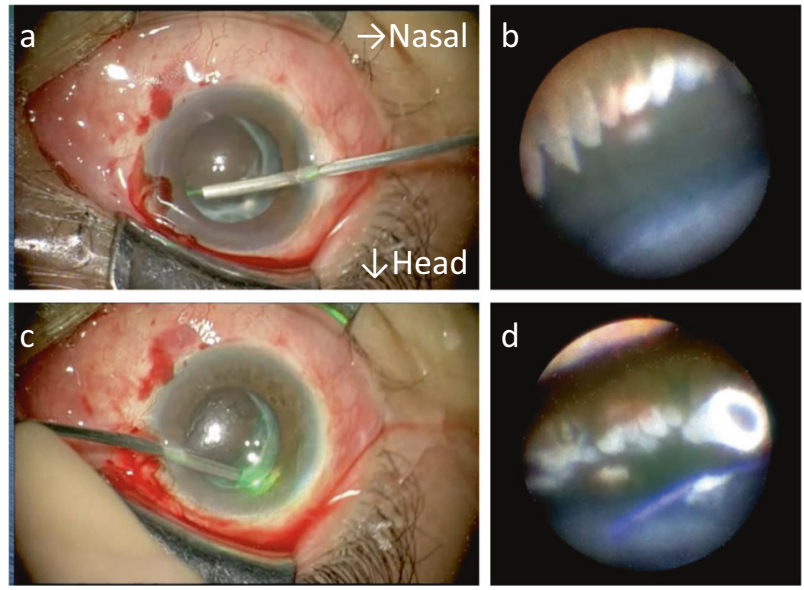

Fig. 2 Intraoperative findings during endoscopic cyclophotocoagulation (ECP) (case 8, right eye). The video captures images from a, $\mathbf{c}$ the surgical microscope and $\mathbf{b}$, $\mathbf{d}$ the endoscope during ECP. ECP is applied around the entire circumference through corneal side ports created $\mathbf{a}, \mathbf{b}$ temporally and $\mathbf{c}, \mathbf{d}$ nasally. $\mathbf{b}, \mathbf{d}$ The coagulated ciliary body surface is white

\section{Measurements}

The clinical parameters, including age, sex, glaucoma type, ocular surgical history, preoperative and postoperative best-corrected visual acuities (BCVAs), IOP, number of antiglaucoma medications, use of oral carbonic anhydrase inhibitors (CAIs), and duration of the postoperative followup were collected from the medical charts. The decimal VA was converted into the logarithm of the minimum angle of resolution (LogMAR) VA. According to a previous study, counting fingers, hand motions, light perception, and no light perception were regarded as decimal VAs of 0.0025 , $0.002,0.0016$, and 0.0013 , respectively [12]. The IOP was measured by Goldmann applanation tonometry except for that measured on postoperative day 3 using the iCARE rebound tonometer (M.E. Technica, Tokyo, Japan). The extent of the ECP, perioperative complications, interventions for complications, and additional glaucoma surgeries performed were collected by reviewing the medical and surgical records.

\section{Statistical analysis}

The preoperative and final VAs, IOPs, and numbers of antiglaucoma medications were compared using paired $t$ test. To adjust for differences in the follow-up periods, the preoperative IOP and IOPs measured at 3 days, 1-2 weeks, 1 month (3-5 weeks), 3 months (2-4 months), 6 months (5-7 months), 9 months ( $8-10$ months), 12 months (11-13 months), 18 months (16-20 months), and 24 months (22-26 months) postoperatively were compared using a mixed-effects regression model in which each patient's identification number was regarded as a random effect and the time period was regarded as a fixed effect, followed by the post hoc comparison against preoperative values using $t$ test. The postoperative changes in the numbers of antiglaucoma medication also were assessed using the mixedeffects regression model. All continuous data are expressed as the mean $\pm \mathrm{SD}$. All statistical analyses were performed using the JMP version 11.0 statistical software (SAS Institute, Inc., Cary, NC, USA). $P<0.05$ was considered statistically significant.

\section{Results}

In this case series, seven eyes had primary open-angle glaucoma (POAG) and three eyes had glaucoma secondary to intracapsular cataract extraction including two eyes with congenital cataract (cases 2 and 5), all of which were treated with ECP (Table 1). Other than cataract surgery, the previous history of ocular surgeries included long-tube shunt surgery (7 eyes), glaucoma filtration surgery (trabeculectomy, ExPRESS shunt (Alcon), or non-penetrating trabeculectomy) (6 eyes), pars plana vitrectomy (6 eyes), trabeculotomy (3 eyes), scleral buckling ( 1 eye), and cyclotherapy (1 eye). Overall, the eyes had undergone a 
Table 2 Preoperative and postoperative IOPs and medications

\begin{tabular}{lllllr}
\hline Time point & No. of eyes & IOP $(\mathrm{mm} \mathrm{Hg})$ & $p$ Value $^{\mathrm{b}}$ & Medication (range) & $p$ Value $^{\mathrm{b}}$ \\
\hline Preoperative & 10 & $27.3 \pm 5.4(23.5-31.1)$ & & $4.4 \pm 0.8(3.8-5.0)$ & \\
3 days & 10 & $9.3 \pm 3.2(7.0-11.6)$ & $<0.0001$ & $2.0 \pm 1.4(1.0-3.0)$ & 0.0016 \\
$1-2$ weeks & 10 & $15.4 \pm 5.7(11.3-19.5)$ & 0.0003 & $2.1 \pm 1.4(1.1-3.1)$ & 0.0016 \\
1 month & 10 & $16.9 \pm 6.8(12.0-21.8)$ & 0.0101 & $1.5 \pm 1.1(0.7-2.3)$ & $<0.0001$ \\
3 months & 10 & $14.8 \pm 4.1(11.9-17.7)$ & 0.0006 & $2.5 \pm 1.6(1.4-3.6)$ & 0.0012 \\
6 months & 10 & $14.3 \pm 3.3(11.9-16.7)$ & 0.0001 & $2.6 \pm 1.6(1.4-3.8)$ & 0.0019 \\
9 months & 10 & $13.2 \pm 6.3(8.7-17.7)$ & 0.0018 & $2.8 \pm 1.3(1.9-3.7)$ & 0.0011 \\
12 months & 10 & $12.4 \pm 6.8(7.6-17.2)$ & 0.0008 & $3.3 \pm 0.9(2.6-4.0)$ & 0.0032 \\
18 months & 6 & $12.8 \pm 5.2(7.4-18.3)$ & 0.0065 & $3.5 \pm 1.0(2.4-4.6)$ & 0.0127 \\
24 months & 4 & $11.5 \pm 6.5(1.2-21.8)$ & 0.0179 & $3.3 \pm 1.0(1.7-4.8)$ & 0.0138 \\
& & $p<0.0001^{\mathrm{a}}$ & & $p<0.0001^{\mathrm{a}}$ & \\
\hline
\end{tabular}

Data are expressed as means \pm standard deviations (upper and lower $95 \%$ confidence intervals)

$I O P$ intraocular pressure

${ }^{\mathrm{a}} p$ Values are calculated using the mixed-effects regression model

${ }^{\mathrm{b}} p$ Values are calculated using paired $t$ test to compare the preoperative and respective time period values mean of $4.3 \pm 1.7$ (range, $2-7$ ) ocular surgeries before ECP. The procedure included ECP alone in six eyes, ECP combined with anterior vitrectomy in three eyes (cases 2, 3, and 4 ), and ECP combined with cataract surgery in one eye (case 1).

The mean preoperative IOP of $27.3 \pm 5.4 \mathrm{~mm} \mathrm{Hg}$ (range, 19-36) and number of antiglaucoma medications of $4.4 \pm$ 0.8 (range, 3-6) decreased significantly $(p=0.0005$ and $p=0.0043$, respectively) to $12.9 \pm 5.2 \mathrm{~mm} \mathrm{Hg}$ (range, $8-20$ ) and $2.7 \pm 1.4$ (range, $0-4$ ), respectively, at the final postoperative visit at $636 \pm 225$ days (range, 350-1058) (Table 1). At the final visit, all eyes achieved IOP control of $\leq 20 \mathrm{~mm} \mathrm{Hg}$ with fewer medications than preoperatively (Table 1). Six patients required oral CAIs preoperatively, while three patients required them postoperatively, including one patient who required CAIs to treat the fellow eye (case 7) (Table 1). The mixed-effects regression model showed that the postoperative decreases in the IOP $(p<$ 0.0001 for the model) and the number of antiglaucoma medications $(p<0.0001$ for the model $)$ were significant (Table 2). Compared with preoperative values, the IOP decreases were significant $(p<0.0001-0.0179)$ at every time point up to 24 months postoperatively. Compared with preoperative values, the decreases in the number of antiglaucoma medications were significant $(p<$ 0.0001-0.0138) up to 24 months (Table 2).

No remarkable complication was recorded during the procedure. The early postoperative complications ( $\leq 3$ months post-ECP) included IOP spikes exceeding 30 $\mathrm{mm} \mathrm{Hg}$ in two eyes (cases 4 and 5) and residual lens cortex in the anterior chamber in one eye (case 1). The late postoperative complications ( $>3$ months post-ECP) included transient choroidal detachment in two eyes (cases 3 and 7), increase of preexisted corneal opacity of unknown aetiology in one eye (case 2), exposure of an Ahmed Glaucoma Valve (JFC Sales Plan Co., Ltd., Tokyo, Japan) in one eye (case 4 ), and pigment dispersion in the anterior chamber in one eye (case 7). The interventions required to treat the complications or additional glaucoma surgery included removal of residual lens cortex at 1 day postoperatively in one eye (case 1), implantation of an Ahmed Glaucoma Valve (model FP-7) at 16 days postoperatively due to an IOP spike in one eye (case 4), and ologen patch graft (model 862051, Aeon Astron Europe B.V., Leiden, The Netherlands) [13] to treat exposure of an Ahmed Glaucoma Valve tube in one eye (case 4). At the final visit, compared with preoperative values, the BCVA decreased in three eyes by $>0.2$ LogMAR unit because of glaucoma progression (case 1, decimal VA of 0.01 preoperatively to hand motions at the final visit; case 3, 0.01 to hand motions; and case 4, 0.02 to 0.01 ); the BCVA remained unchanged in seven eyes.

\section{Representative case}

A 62-year-old man (case 1) had undergone multiple glaucoma surgeries including a trabeculotomy ab externo, two trabeculectomies combined with intraoperative use of mitomycin C, and three implantations of a Ahmed Glaucoma Implant (model PC-7 in the inferotemporal and inferonasal quadrants and model FP-7 in the superotemporal quadrant) to treat POAG in his right eye (Fig. 3). The BCVA and IOP were 0.01 (decimal VA) and $30 \mathrm{~mm} \mathrm{Hg}$, respectively, with three topical antiglaucoma medications and an oral CAI. ECP was applied to three fourths of the circumference in combination with small incision cataract surgery in the right eye. A marked IOP decrease to $<10 \mathrm{~mm}$ $\mathrm{Hg}$ occurred 1 day postoperatively and was sustained for 4 months; the IOP then increased to around $15 \mathrm{~mm} \mathrm{Hg}$ and 

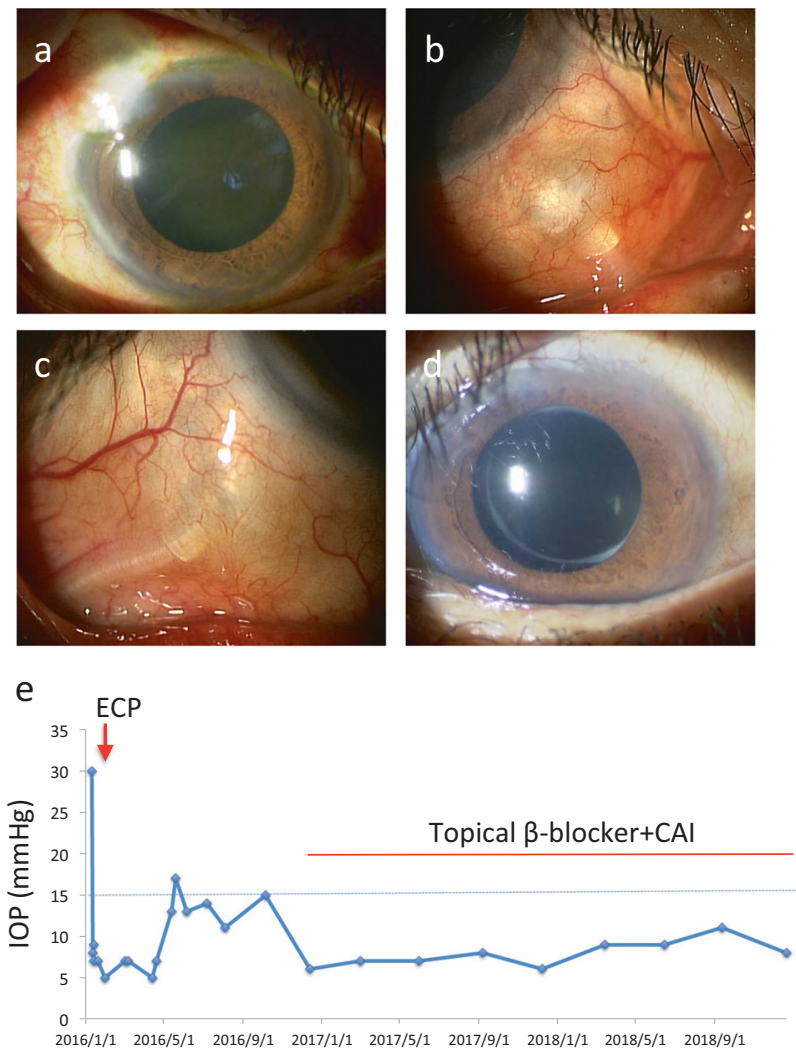

Fig. 3 Representative case (case 1, right eye). The patient had undergone multiple glaucoma surgeries including one trabeculotomy ab externo, two trabeculectomies, and three Ahmed Glaucoma Implants to treat primary open-angle glaucoma in his right eye. Before endoscopic cyclophotocoagulation (ECP), a conjunctival scarring around the entire circumference of the corneal limbus, previously implanted tube shunts $\mathbf{b}$ in the inferotemporal quadrant and $\mathbf{c}$ in the inferonasal quadrant are seen by slit-lamp examination. $\mathbf{d}$ One month after ECP, no marked inflammation of the ocular surface or anterior chamber is seen. e The intraocular pressure is normal after ECP for at least 35 months postoperatively. CAI carbonic anhydrase inhibitor

was sustained for 5 months (Fig. 3). After the start of a topical $\beta$-blocker and CAI fixed-combination eye drop, the IOP remained $<10 \mathrm{~mm} \mathrm{Hg}$ until the last follow-up visit. At the last follow-up visit at 35 months postoperatively (Fig. 3), the BCVA and IOP were hand motions and $9 \mathrm{~mm}$ $\mathrm{Hg}$, respectively, with two antiglaucoma medications. Residual lens cortex in the anterior chamber was removed 1 month postoperatively, otherwise, no surgical complication or additional intervention was related to the surgery in this case.

\section{Discussion}

In the current case series, after one ECP procedure with a $532 \mathrm{~nm} \mathrm{Nd:YAG} \mathrm{laser} \mathrm{in} \mathrm{glaucomatous} \mathrm{eyes} \mathrm{that} \mathrm{had} \mathrm{been}$ treated previously with a mean of 4.3 ocular surgeries, the IOP decreased by $14.4 \mathrm{~mm} \mathrm{Hg}$ (53\% reduction) with 1.7
(39\% reduction) fewer antiglaucoma medications at the mean final follow-up visit at 21 months postoperatively. The surgical efficacy seen in the current study seemed comparable to previous ECP studies using an 810-nm diode laser. Uram, who developed the ECP procedure, reported in the initial case series of ECP that included 10 patients with intractable neovascular glaucoma that the IOP decreased by $28.3 \mathrm{~mm} \mathrm{Hg}$, a $65 \%$ reduction, at the mean final follow-up examination at 8.8 months postoperatively [9]. A subsequent study performed in the United States included 68 eyes treated with maximal medical therapy and failed trabeculectomy or TSCP or both; after diode laser ECP was applied to $180^{\circ}-360^{\circ}$ of the ciliary body circumference, the IOP decreased by a mean of $10.7 \mathrm{~mm} \mathrm{Hg}$, a $39 \%$ reduction, at the final follow-up examination at 12.9 months postoperatively [14]. A Chinese study of 51 eyes with refractory glaucoma treated with diode laser ECP reported that the IOP decreased a mean of $18.3 \mathrm{~mm} \mathrm{Hg}$, a $52 \%$ reduction, with fewer antiglaucoma medications at 24 weeks postoperatively [15]. An Indian study of 50 eyes refractory to medical and surgical interventions reported that, after treatment with diode laser ECP, the IOP decreased a mean of $18.6 \mathrm{~mm} \mathrm{Hg}$, a $57 \%$ reduction, with $1.4(57 \%)$ fewer antiglaucoma medications at 12.3 months postoperatively [16]. At the final visit, all current eyes achieved IOP control of $\leq 20 \mathrm{~mm} \mathrm{Hg}$ with fewer medications than preoperatively; three of the six patients discontinued oral CAIs. Thus ECP with $532 \mathrm{~nm}$ laser seems effective for normalizing the IOP even in Japanese eyes with refractory glaucoma.

Autopsy studies have shown that as little as $35 \%$ of the diode laser energy emitted on the scleral surface reaches the pigmented epithelium [17]. Accordingly, TSCP requires more overall power, which induces more inflammation than ECP [11]. In cadaver eyes, diode laser TSCP induced coagulation necrosis of both the ciliary body epithelium and stroma [18]. In porcine eyes, TSCP-treated tissue showed pronounced tissue disruption of the ciliary body muscle and stroma, ciliary processes, and both pigmented and nonpigmented ciliary epithelium, while ECP-treated tissue exhibited pronounced contraction of the ciliary processes with disruption of the ciliary body epithelium with less architectural disorganization and sparing of the ciliary body muscle [19]. In rabbit eyes, compared with post-ECP, fluorescein angiography and histopathology confirmed the chronic poor perfusion and greater vascular occlusion of the ciliary body seen in eyes after TSCP [20]; vascular occlusion may account for significant complications including hypotony and phthisis after TSCP. Previous studies have shown that, compared with TSCP, diode laser ECP appears to be a more selective form of cyclophotocoagulation resulting in less tissue disruption that achieves the goal of destroying ciliary body epithelium [19]. Use of a shorter wavelength of the laser source (532 nm Nd:YAG) than that 
of previous studies (810-nm diode) might be associated with more selective surface ablation of the ciliary epithelium and leave the deep tissue unaffected, but this needs to be tested. A rabbit study showed that the ciliary body blood flow decreased immediately after the ECP procedure, after which some reperfusion at 1 week and greater reperfusion by 1 month were seen [20]. This agrees well with the lowest IOP at 3 days and elevation of the IOP 1-2 weeks postoperatively in the current cases (Table 2).

Early postoperative complications included IOP spikes in two cases, while no marked inflammation occurred. African-American compared with Caucasian ethnicity was associated significantly with postoperative anterior chamber reactions after diode laser ECP [21]; thus Asian patients may have less ECP-related inflammation compared with African-American patient, but this needs confirmation. Use of triamcinolone acetonide at the end of surgery in all cases can be another explanation for the lower incidence of inflammation-related complications in the current case series. Regarding late postoperative complications, transient choroidal detachment and pigment dispersion recorded are known complications of diode laser ECP [22]. During the mean follow-up of 21 months, no catastrophic surgical complications such as phthisis bulbi or persistent hypotony developed in the current cases. Previous studies have reported that hypotony and phthisis bulbi developed less frequently with diode laser ECP ( $\sim 8 \%$ and $\sim 3 \%$, respectively) [22] compared to diode laser TSCP ( 25\% and $\sim 9.9 \%$, respectively) [10]. Although three eyes had a BCVA that decreased by $>0.2 \operatorname{LogMAR}$ unit, these cases were all end-stage cases, and the visual decreases were related to glaucoma progression rather than complications. Thus the safety profile of our case series seems comparable to or even better than previous reports and was considered acceptable considering the refractory glaucoma being treated. High ECP powers of 500-900 $\mathrm{mW}$ have been associated with serious complications, such as fibrin exudates (24\%), hyphema (12\%), hypotony (8\%), and visual loss (6\%) [14]. Subsequent studies with powers $<500 \mathrm{~mW}$ tended to be associated only with inflammation and hyphema without a drastic decrease in the success rate [22]. Laser energy and tissue damage depend on the distance between the probe and target, instead of wattage; however, no study to date has confirmed increased efficacy with a shorter laser distance. A previous study of the Endo Optiks device recommended that the optimal distance between the treated tissue and laser probe was $2 \mathrm{~mm}$ and that the probe be kept at a distance at which six ciliary processes were visualized on the video monitor [23]. Use of the probe with a focus distance of $>2 \mathrm{~mm}$ and with lower laser power of $150-200 \mathrm{~mW}$ in our procedure may be associated with the safety profile in the current case series.
At 12 months postoperatively, ECP normalized $(\leq 21 \mathrm{~mm}$ $\mathrm{Hg}$ ) the IOP in $88 \%$ of eyes that had undergone previous failed tube shunt surgeries [24]. Both ECP and implantation of a second glaucoma drainage device were equally effective as second surgeries for refractory glaucoma that had been treated previously with a tube shunt surgery [25]. A meta-analysis showed that the efficacy of ECP for refractory glaucoma was comparable to non-ECP treatments, including trabeculectomy, Ahmed Glaucoma Valve implantation, TSCP, and cyclocryotherapy [26]. Before ECP, the current cases had undergone multiple filtration/tube shunt surgeries or difficult-to-perform filtration/tube shunt surgeries because of scleral thinning (case 2) or extensive conjunctival scarring (cases 3-5). Currently, no specialized device for ECP is approved in Japan. Although the ECP probe was a prototypic, both the endocopy and laser devices used have been approved as vitreoretinal surgery devices in Japan and many other countries. Collectively, we concluded that reduced aqueous production resulting from $532 \mathrm{~nm}$ laser ECP is a reasonable option when multiple aqueous drainage surgeries have failed or when a scleral/conjunctival flap was difficult to create.

\section{Summary}

\section{What was known before:}

- Endoscopic cyclophotocoagulation with $810 \mathrm{~nm}$ diode laser is effective to reduce intraocular pressure in a wide range of glaucoma, including refractory glaucoma and early-to-moderate-stage glaucoma.

\section{What this study adds:}

- Endoscopic cyclophotocoagulation with $532 \mathrm{~nm}$ neodymium-doped yttrium aluminium garnet $(\mathrm{Nd}$ : YAG) laser is effective to reduce intraocular pressure in Japanese patients with refractory glaucoma.

Acknowledgements The authors acknowledge Mr. Yosuke Yoshimoto and Mr. Koji Yamada (FiberTech Co., Ltd., Chiba, Japan) for their collaborative work and technical support in developing the ECP device.

\section{Compliance with ethical standards}

Conflict of interest The endoscopic cyclophotocoagulation device was co-developed by MT and FiberTech Co., Ltd., Chiba, Japan. The device used in this study was provided by FiberTech Co., Ltd. The other authors declare that they have no conflict of interest.

Publisher's note: Springer Nature remains neutral with regard to jurisdictional claims in published maps and institutional affiliations. 


\section{References}

1. Garway-Heath DF, Crabb DP, Bunce C, Lascaratos G, Amalfitano $\mathrm{F}$, Anand $\mathrm{N}$, et al. Latanoprost for open-angle glaucoma (UKGTS): a randomised, multicentre, placebo-controlled trial. Lancet. 2015;385:1295-304.

2. Tanito M, Ikeda Y, Fujihara E. Effectiveness and safety of combined cataract surgery and microhook ab interno trabeculotomy in Japanese eyes with glaucoma: report of an initial case series. Jpn J Ophthalmol. 2017;61:457-64.

3. Tanito M, Sugihara K, Hara K, Takai Y. Different glaucoma types and glaucoma surgeries among different age groups. Graefe's Arch Clin Exp Ophthalmol. 2018;256:2013-4.

4. Bietti G. Surgical intervention on the ciliary body; new trends for the relief of glaucoma. JAMA. 1950;142:889-97.

5. Lin P, Wollstein G, Glavas IP, Schuman JS. Contact transscleral neodymium:yttrium-aluminum-garnet laser cyclophotocoagulation Long-term outcome. Ophthalmology. 2004;111:2137-43.

6. Seibold LK, SooHoo JR, Kahook MY. Endoscopic cyclophotocoagulation. Middle East Afr J Ophthalmol. 2015;22:18-24.

7. Patel A, Thompson JT, Michels RG, Quigley HA. Endolaser treatment of the ciliary body for uncontrolled glaucoma. Ophthalmology. 1986;93:825-30.

8. Zarbin MA, Michels RG, de Bustros S, Quigley HA, Patel A. Endolaser treatment of the ciliary body for severe glaucoma. Ophthalmology. 1988;95:1639-48.

9. Uram M. Ophthalmic laser microendoscope endophotocoagulation. Ophthalmology. 1992;99:1829-32.

10. Ishida K. Update on results and complications of cyclophotocoagulation. Curr Opin Ophthalmol. 2013;24:102-10.

11. Kaplowitz K, Kuei A, Klenofsky B, Abazari A, Honkanen R. The use of endoscopic cyclophotocoagulation for moderate to advanced glaucoma. Acta Ophthalmol. 2015;93:395-401.

12. Grover S, Fishman GA, Anderson RJ, Tozatti MS, Heckenlively JR, Weleber RG, et al. Visual acuity impairment in patients with retinitis pigmentosa at age 45 years or older. Ophthalmology. 1999;106:1780-5.

13. Tanito M, Sano I, Ikeda Y, Fujihara E. Patch grafting using an ologen collagen matrix to manage tubal exposure in glaucoma tube shunt surgery. Case Rep Ophthalmol. 2018;9:9-16.

14. Chen J, Cohn RA, Lin SC, Cortes AE, Alvarado JA. Endoscopic photocoagulation of the ciliary body for treatment of refractory glaucomas. Am J Ophthalmol. 1997;124:787-96.

15. Yu MB, Huang SS, Ge J, Guo J, Fang M. [The clinical study of endoscopic cyclophotocoagulation on the management of refractory glaucoma]. Zhonghua Yan $\mathrm{Ke} \quad \mathrm{Za}$ Zhi. 2006;42:27-31.

16. Murthy GJ, Murthy PR, Murthy KR, Kulkarni VV, Murthy KR. A study of the efficacy of endoscopic cyclophotocoagulation for the treatment of refractory glaucomas. Ind $\mathrm{J}$ Ophthalmol. 2009;57:127-32.

17. Vogel A, Dlugos C, Nuffer R, Birngruber R. Optical properties of human sclera, and their consequences for transscleral laser applications. Lasers Surg Med. 1991;11:331-40.

18. Assia EI, Hennis HL, Stewart WC, Legler UF, Carlson AN, Apple DJ. A comparison of neodymium: yttrium aluminum garnet and diode laser transscleral cyclophotocoagulation and cyclocryotherapy. Invest Ophthalmol Vis Sci. 1991;32:2774-8.

19. Pantcheva MB, Kahook MY, Schuman JS, Rubin MW, Noecker RJ. Comparison of acute structural and histopathological changes of the porcine ciliary processes after endoscopic cyclophotocoagulation and transscleral cyclophotocoagulation. Clin Exp Ophthalmol. 2007;35:270-4.

20. Lin SC, Chen MJ, Lin MS, Howes E, Stamper RL. Vascular effects on ciliary tissue from endoscopic versus transscleral cyclophotocoagulation. Brit J Ophthalmol. 2006; 90:496-500.

21. Edmiston AM, SooHoo JR, Seibold LK, Kahook MY, Palestine AG, Pantcheva MB. Postoperative inflammation after endoscopic cyclophotocoagulation: racial distribution and effect on outcomes. J Glaucoma. 2018;27:266-8.

22. Cohen A, Wong SH, Patel S, Tsai JC. Endoscopic cyclophotocoagulation for the treatment of glaucoma. Surv Ophthalmol. 2017;62:357-65.

23. Yu JY, Kahook MY, Lathrop KL, Noecker RJ. The effect of probe placement and type of viscoelastic material on endoscopic cyclophotocoagulation laser energy transmission. Ophthalmic Surg Lasers Imaging. 2008;39:133-6.

24. Francis BA, Kawji AS, Vo NT, Dustin L, Chopra V. Endoscopic cyclophotocoagulation (ECP) in the management of uncontrolled glaucoma with prior aqueous tube shunt. $\mathrm{J}$ Glaucoma. 2011;20:523-7.

25. Murakami Y, Akil H, Chahal J, Dustin L, Tan J, Chopra V, et al. Endoscopic cyclophotocoagulation versus second glaucoma drainage device after prior aqueous tube shunt surgery. Clin Exp Ophthalmol. 2017;45:241-6.

26. Yang Y, Zhong J, Dun Z, Liu XA, Yu M. Comparison of efficacy between endoscopic cyclophotocoagulation and alternative surgeries in refractory glaucoma: a meta-analysis. Medicine. 2015;94:e1651. 